\title{
Humans' Ability to Assess Emotion in Dog Barks Only Slightly Affected by their Country of Residence, a Replication of Pongrácz et al. (2005) in a Portuguese Sample
}

\author{
Karine Silva ${ }^{1, \#, ~ T a m a ́ s ~ F a r a g o ́ n o, \#, *, ~ P e ́ t e r ~ P o n g r a ́ c z ~}{ }^{2}$, Patrícia Romeiro, \\ Mariely Lima ${ }^{3}$, and Liliana de Sousa ${ }^{1}$
}

\author{
${ }^{1}$ Instituto de Ciências Biomédicas Abel Salazar, Departamento de Ciências do Comportamento, Porto, Portugal. \\ ${ }^{2}$ Department of Ethology, Eötvös Loránd University, Budapest, Hungary \\ ${ }^{3}$ Escola Superior de Saúde da Universidade de Aveiro, Portugal \\ ${ }^{\#}$ Both authors contributed equally to this work \\ *Corresponding author (Email: mustela.nivalis@gmail.com)
}

Citation - Silva, K., Faragó, T., Pongrácz, P., Romeiro, P., Lima, M., \& de Sousa, L. (2021). Humans' ability to assess emotion in dog barks only slightly affected by their country of residence, a replication of Pongrácz et al. (2005) in a Portuguese sample. Animal Behavior and Cognition, 8(2), 107-123. https://doi.org/10.26451/abc.08.02.02.2021

\begin{abstract}
An original experiment by Pongrácz et al. (2005) showed that Hungarian listeners were able to categorize recordings of dog barks recorded in various contexts above chance level and assessed the barking dogs' emotional state in accordance with these contexts. The present study sought to replicate this experiment in a Portuguese sample. Similar to Hungarians, Portuguese dog owners and non-owners rated the barks in accordance with their specific social contexts (e.g., a dog left alone is most probably in fearful state instead of a playful one), and correctly categorized territorial and separation barks above chance level. Similar error patterns were observed across subjects from the two countries. The Portuguese participants, however, were less successful in categorizing barks than the Hungarians. Additionally, results showed both sex and dog ownership effects. Women from both countries tended to attribute higher levels of despair to all bark recordings. Moreover, within the Portuguese sample, dog-owners were generally more accurate in categorizing the recordings than non-owners. Overall, the results of our replication study, besides supporting the universality of vocal emotion recognition in mammals, calls attention to the possibility of country/culture- and also individual-specific effects on human perception and understanding of nonhuman vocalizations.
\end{abstract}

Keywords - Acoustic communication, Bark, Cultural differences, Domestic dog, Emotion

Affective communication, which means the signaling of the emotional state of the caller has a crucial role in social interactions across the animal kingdom. Affective communication makes it possible through assessing others' inner state to foretell their subsequent behavior actions and react adaptively. Humans are particularly good at reading others' behavior; however, their ability to recognize emotions in vocalizations is not restricted to intra-specific communication but also appears relevant in interactions with other, nonhuman animals (dogs: Faragó et al., 2017; cats: Nicastro \& Owren, 2003; pigs: Tallet et al., 2010; but see also studies in macaques: Linnankoski et al., 1994; and various species Filippi et al., 2017). On one hand, the ability to recognize emotions of other species might be the result of the common roots of the neural background of emotions and voice production and the act of the simple rules of vocal emotion encoding (Andics \& Faragó, 2019). On the other hand, ample evidence suggests the adaptivity of processing 
and understanding emotions in heterospecific calls and reacting to them adequately to avoid predators (Fallow \& Magrath, 2010; Kitchen et al., 2010; Magrath et al., 2015) or find food (Koda, 2012). This ability might have been adaptive and provided extra benefit for humans during the process of domesticating animals. In the course of the domestication of any species, especially ones having the abilities to pose a fatal threat for early humans like early dogs, successful reading of the emotional states of individuals might have been crucial. Humans' perception and understanding of dog barks, in particular, is being extensively explored, with available data suggesting that barks serve as an effective means of communication between the two species (for reviews see Pongrácz, 2017; Pongrácz et al., 2010).

In an original study, Pongrácz et al. (2005) showed that Hungarian listeners, irrespective of their experience with dogs, were able to associate emotional ratings to contextually variable vocalizations of a Hungarian herding dog breed, the Mudi. This breed was chosen in the original study due to its tendency to extensively bark in various contexts. Findings from a subsequent study (Pongrácz et al., 2006) indicated that, when rating the inner states of dogs, Hungarian listeners were relying on basic acoustic parameters of dog barks as would be predicted by Morton's 'structural motivational' rules that apply universally to the affective vocalizations in birds and mammals: deep pitched barks with fast repetition pulsing were mostly considered aggressive and fearless, whereas high pitched, slowly repeating, rough (atonal) barks tended to be considered as happy or playful, and finally, high-pitched, slowly repeating, clear (tonal) barks were generally evaluated as being fearful and desperate (Pongrácz et al., 2006).

Additionally, Pongrácz et al. (2005) showed that participants could accurately categorize the bark samples according to the context in which they were produced. That is, listeners not only perceived emotions in the dog barks (as evidenced by the emotional ratings attributed to the recordings) but also recognized their context. Interestingly, in this study, Mudi owners (i.e., participants who owned a Mudi at the time of the experiment or earlier) were only slightly better at categorizing the barks than other dog owners (i.e., participants who owned a dog at the time of the experiment or earlier, but never owned a Mudi) and non-dog owners (i.e., participants who never owned a dog at home), with no significant between-group differences observed. Also, in another study, Molnár et al. (2010) found that even congenitally sightless Hungarian individuals as well as children as young as 10 years old (Pongrácz et al., 2011) who never owned a dog, perceived emotions in the bark samples and categorized them appropriately.

Taken together, the above studies suggest that humans' ability to perceive emotions in dog barks and to extract context-specific information from these vocalizations may be shaped more by universal, cross-taxa acoustic coding and processing mechanisms that support emotional communication both within and across species, than by experience-dependent, higher-order cognitive processes. Indeed, recent evidence indicates that humans' rate conspecific emotional vocalizations along basic acoustic rules and apply similar rules when processing dog vocalizations (Faragó et al., 2014). This would be in line with the idea that humans utilize similar mechanisms for recognizing con- and heterospecific vocal emotions (Andics et al., 2014). The referred line of investigation, however, has limited generalizability as only Hungarian listeners were considered. Thus, to this point, potential effects of culture- and country-related factors on human perception and understanding are left unexplored. Attitudes towards dogs, for example, might well differ across countries and cultures (Miura et al., 2000; Turcsán et al., 2012) and nothing is known of whether such differences may impact on human-dog emotional communication.

Also, nothing is yet known on the extent of universality versus cultural/country-specificity across the two emotional domains of perception and understanding of dog barks. In the case of intra-specific communication, it has been suggested that, when compared to understanding emotions, emotional perception either through facial cues or vocalizations may be more 'universal' (i.e., rooted in evolutionary processes that determine the decoding and production of emotional expressions), with only minor variation across cultures lcountries, due to slight differences in display rules, decoding rules, or culture-specific affect programs (e.g., Ekman et al., 1987; Elfenbein, 2006; Elfenbein \& Ambady, 2002; Elfenbein et al., 2002). Conversely, understanding emotions may be more heavily influenced by culture, due to dissimilarities in emotional concepts, preferences, and vocabulary, as well as interpretation of emotional triggers (Matsumoto \& Assar, 1992; Matsumoto \& Hwang, 2012; Scherer, 1997), which are "socially constructed to reflect the frequency and importance of events and emotions within the cultural system" (Shao et al., 2014, p. 234). 
To the best of our knowledge, no attempt has yet been made to test for these propositions within the particular case of inter-species communication.

Clearly, the replication of Pongrácz et al.'s (2005) original study among different countries/cultures would offer an interesting opportunity to do so. The present study aims at taking a first and preliminary step in this direction by reproducing the exact methods used in Pongrácz et al. on a convenience sample of Portuguese individuals matched in age, gender and experience with dogs with the original Hungarian sample (unfortunately, there were no further details available about the demographic composition of the original sample).

If, as suggested in the literature, emotion perception is country/culture independent (being a biologically rooted process based on the same rules across species), then we expected to find no significant differences between data obtained here and that reported for Hungarian listeners. Conversely, and considering that context recognition, by being based on higher level cognitive processes that can be more affected by culture, including general attitudes towards dogs and dog barks, we predicted significant differences in successful recognition of the social context of the barks between the Portuguese listeners here and the results of the Hungarians from the original study (Pongrácz et al., 2005).

\section{Method}

The method used in this study was an exact replication of the original paper using the same (bark sound) recordings and a Portuguese translation of the questionnaires (details can be found in Pongrácz et al., 2005).

\section{Participants}

A total of 51 individuals, none of whom had prior contact with a Mudi, participated. Two experimental groups were formed, based on participants' general experiences with dogs: dog owners, i.e., individuals who owned a dog at the time of the experiment or earlier $\left(n=26 ; M_{\text {age: }} 35.6\right.$ years; range: 18 68 years; men/women: 13/13) and non-owners, i.e., individuals who never owned a $\operatorname{dog}\left(n=25 ; M_{\text {age }} 29.2\right.$ years: range: 19-56 years; men/women: 13/12).

\section{Bark Samples}

Bark samples were recorded from 19 Mudis in six social contexts:

- 'stranger,' when an unknown human male appeared at the garden or at the door of the owner's home;

- 'Schutzhund (or 'fight'),' when the dog was on leash, and a human male acting as a 'bad guy' imitated an attack on the dog (as in Schutzhund training);

- ' 'going for a walk,' when the owner acted like they were preparing for a walk;

- 'alone,' when the owner tethered the dog to a tree in a park and walked out of sight;

- 'ball,' when the owner held a ball or toy and teased the dog with it playfully;

- 'play,' when the owner played with the dog tug-of-war, chasing, wrestling, etc.

In total, 72 barks were used, 12 from each social context originating from different individuals chosen randomly (for additional detail on the contexts and information on recordings and preparation of sound material, see Pongrácz et al., 2005). 


\section{Playback Experiments}

Playback experiments were run at the Institute of Biomedical Sciences Abel Salazar (University of Porto). Each participant was individually presented with a unique set of 18 bark samples including three different barks from each of the six contexts. Samples were prepared in advance and played at the same volume, one-by-one controlled by the experimenter who kept the volume at a level that was comfortable for listeners. Upon request, one repetition of a sample was allowed, and the experimenter moved on to the next playback when the subject finished completing the questionnaire (approximately $30 \mathrm{~s}$ ). Following Pongrácz et al. (2005), no two participants heard exactly the same 18 barks, and the order of the barks within the sets was also randomized. No individual bark was played twice for one participant within one session.

Each listener had to complete two different questionnaires during two separate playback sessions with a short 1-2 min long break, while the experimenter presented the sound material. In the first session (emotionality ratings; questionnaire 1), the participant had to rate each bark sample on a five-point Likert scale for five different inner states: aggressiveness, fearfulness, despair, playfulness, and happiness. Low values indicated the absence of that type of emotion, whereas higher values suggested a predominant presence of the emotion in question.

Following the first session after a short break, the experimenter played the same bark samples once more for the listeners who, at this point of the study, were asked to categorize each bark into one of the possible six social contexts listed on their questionnaire (categorization of the bark samples; questionnaire 2). Employing a forced choice set-up, only those contexts were offered for the participants that the bark samples were originally recorded from. Importantly, the participants were not informed that they were listening to the same samples as in Session 1, nor did they know that each context could occur exactly three times in the 18 -sample set.

\section{Statistical Analyses}

During the playback experiments, each subject heard three barks from each of the six contexts; thus, we averaged the responses within subjects, for each context, both for the emotionality ratings (questionnaire 1) and the number of correct categorization answers (questionnaire 2) and used these values in subsequent analyses.

As ratings were skewed, we applied a Generalized Linear Mixed Models (lme4 package, glmer function) with gamma distribution, identity link and participant ID as random factor. In the case of the success data (context recognition), binomial distribution with logit link was applied. For each model, we used stepwise, backwards elimination-based model selection (drop1) using Likelihood Ratio Tests (LRT) to identify the most parsimonious models. For post-hoc pairwise comparisons we used Tukey method (emmeans).

First, to explore the patterns of how Portuguese subjects rate the emotional content of barks and as an attempt to replicate the original findings, we ran a model selection process from an initial model including emotion scale, bark context and ownership status as main effects and all their two- and three-way interactions.

Then, to directly compare the ratings from Portugal and Hungary we pooled the new dataset with the original one (without the Mudi owners, $n=37, M_{\text {age: }}$ 30.9 years; range: 19-69 years; men/women: 18/18; owner/non-owner: 19/17) and built a second initial model with context, emotion scales and country as main effects and their two- and three-way interactions.

Third, we tested how the gender of the listeners affects the emotional ratings of barks. For this, we built an initial model containing this demographic factor besides the emotion ratings and the context and their two- and three-way interactions.

The same three sets of models were built for the context recognition data, except there was no emotion factor added. 
To analyze the accuracy of context categorization, we first ran a one-sample Wilcoxon signed rank test comparing the participants' answers against the random level (by chance listeners would be correct on 3 out of 18 cases: $16.67 \%$ ). Also, a confusion matrix was created by calculating the ratio of the different contexts given as answers within each original context.

All analyses were run in R (4.0.2) using RStudio (1.3.1073) and a significance level of $p<.05$ was used. In post-hoc tests, corrected $p$ values are reported.

\section{Results}

\section{Replication of Emotion Assessment}

In line with the former Hungarian results, within the Portuguese sample, we found no effect of dogownership, neither as interactions nor as a main effect. After the model selection, only an interaction between the emotional scales and the context of the barks $\left(L R T: \chi^{2}(20)=300.71 ; p<.001\right)$ remained in the parsimonious model. Tukey-adjusted pairwise comparisons revealed a similar pattern as it was described in the original study (Figure 1; Table S1A). On the aggression scale, the contexts 'stranger' and 'Schutzhund' got the highest ratings, differing significantly from every other context. The 'alone' context received the lowest ratings of aggression, although the post-hoc comparison showed a significant difference only from 'play' barks. In case of the fear scale, only 'play' barks received significantly lower ratings than barks from other contexts except 'asking for ball,' while despair showed a similar pattern to fear scale, although 'Schutzhund,' 'before walk' and 'alone' contexts received similarly the highest ratings. On playfulness and happiness scales, the rating pattern again is very similar to each other; 'play' barks received the highest scores, 'before walk,' 'alone' and 'asking for ball' contexts received intermediate scores and 'stranger' and 'Schutzhund' received the lowest ratings.

\section{Figure 1}

The Distribution of Emotion Ratings on the Five Different Scales and the Differences Among the Six Social Contexts
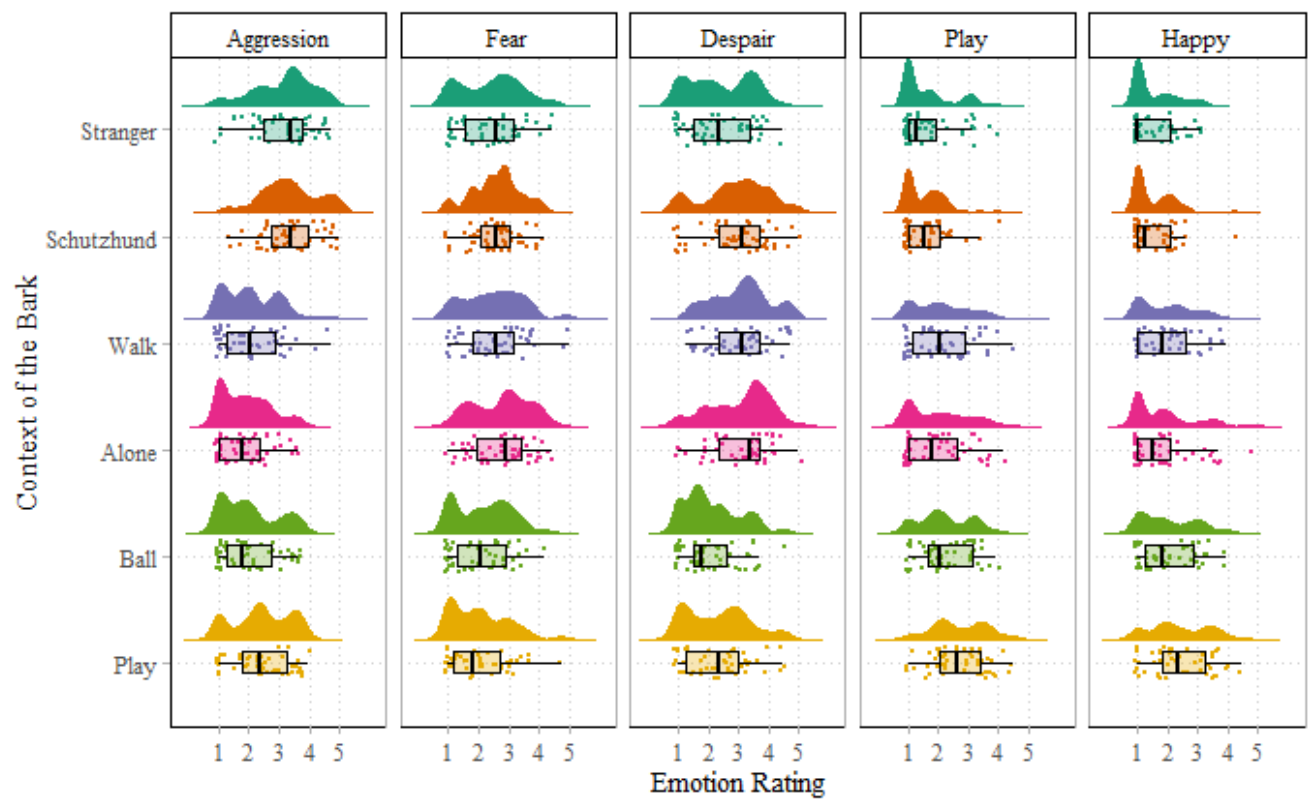

Note. The dots show the actual averaged scores, the boxes show median, 0.25 and 0.75 quartiles and the whiskers show the range. 
Within the 'stranger at fence' context (Figure 2; Table S1B), we found that the aggression ratings were the highest, fear-despair were at medium levels, and playful-happy scales were the lowest. A similar pattern to this occurred in the 'Schutzhund' context but despair ratings were higher and did not differ from the aggression ratings. 'Before walk' barks had the highest ratings on the despair scale, then came the fear, while aggression, playfulness and happiness ratings were the lowest. 'Alone' barks had a similar rating pattern, although fear and despair ratings were both the highest. In the 'asking for ball' context, we found equally low ratings on each scale, no significant difference was found. Finally, in the case of 'play' barks, playfulness received the highest ratings, while aggression, despair and happiness received intermediate, and fear got the lowest scores.

\section{Figure 2}

The Distribution of Emotion Ratings on the Barks from the Six Different Social Contexts and the Differences Among the Five Emotional Scales

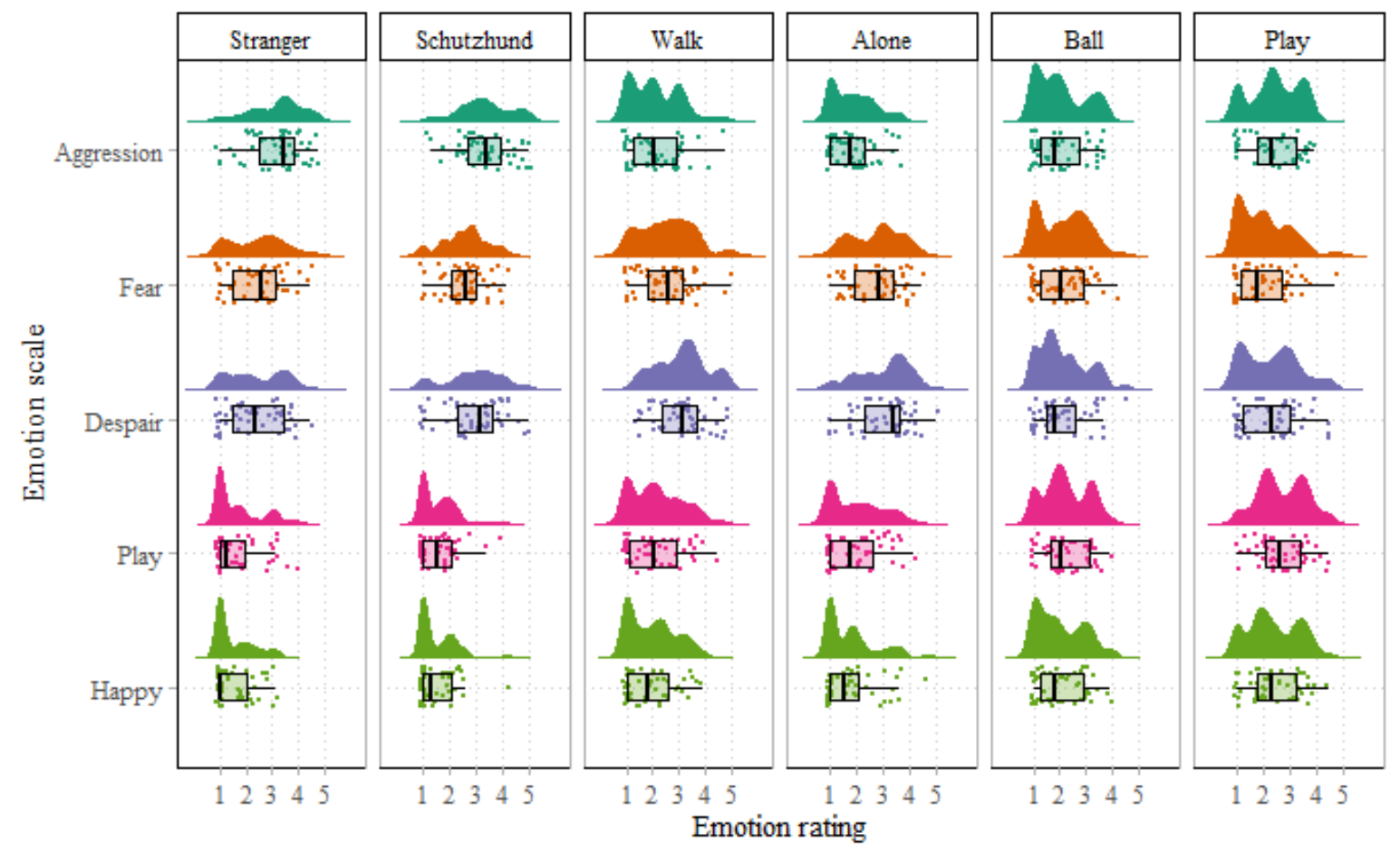

Note. The dots show the actual averaged scores, the boxes show median, 0.25 and 0.75 quartiles and the whiskers show the range.

\section{Difference Between the Portuguese and Hungarian Ratings}

We found that the three-way interaction between country, context and emotion scale was significant $\left(L R T: \chi^{2}(20)=53.31 ; p<.001\right)$. The post-hoc comparisons (Figure 3; Table S1C) revealed that Hungarians rated the aggression higher in both 'stranger' and 'Schutzhund' barks but lower in 'play' barks than Portuguese participants. Fear ratings by the Portuguese were only slightly higher in the 'before walk' barks, but despair ratings were significantly higher in 'stranger,' 'Schutzhund' and 'before walk' contexts. The Hungarians rated playfulness higher than did the Portuguese in 'before walk' and 'play' barks. Finally, Hungarian participants rated 'before walk' barks to be happier. 
Figure 3

Interaction Between the Country of the Listeners, the Context of the Barks and the Emotion Scales

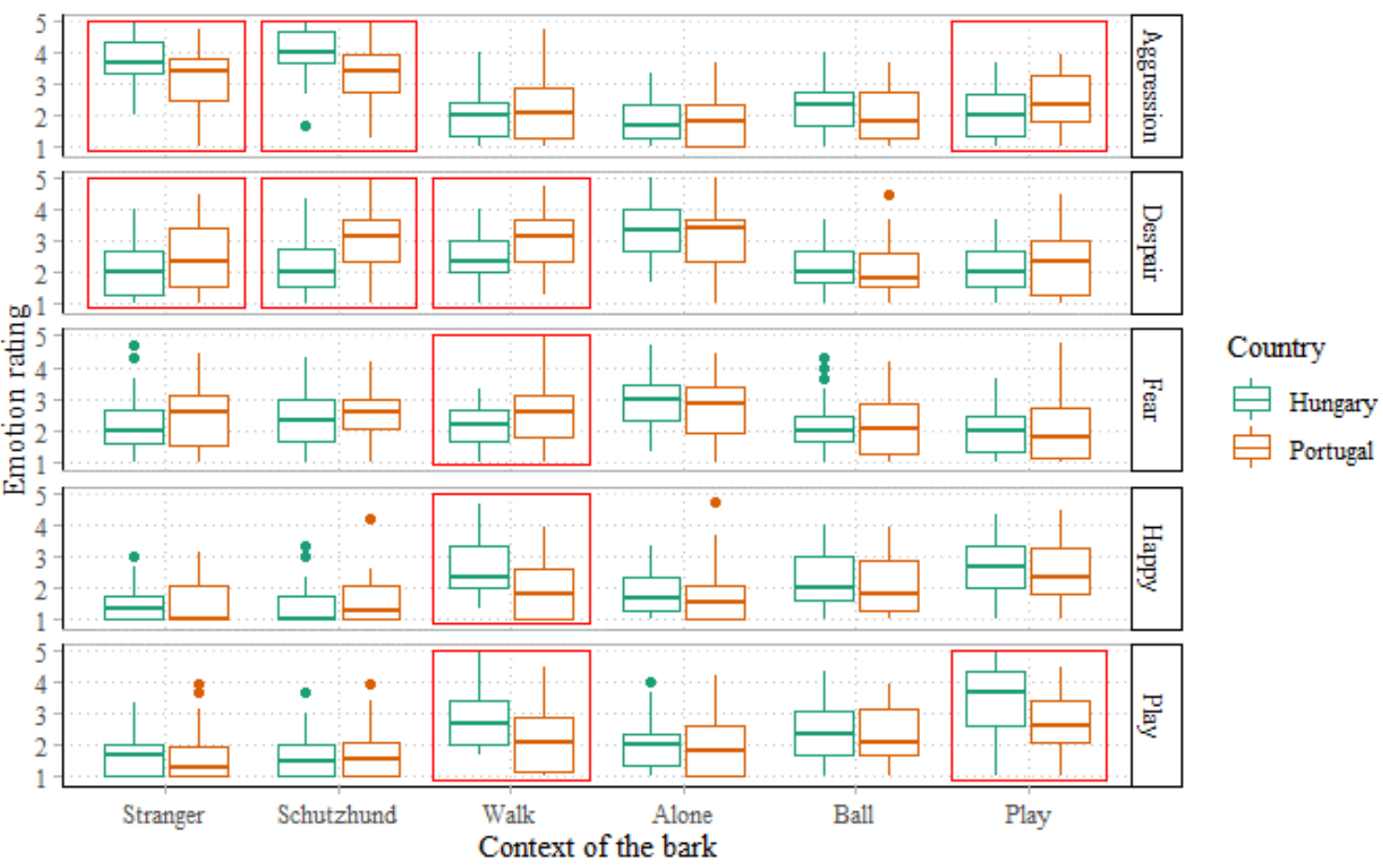

Note. Within the boxplots, the horizontal line represents the median, the box shows the quartiles, the whiskers represent the range, while the circles represent the outliers. The red rectangles highlight the significant differences.

\section{Gender Effect}

We found a gender effect in the emotional ratings $\left(L R T: \chi^{2}(4)=22.88 ; p<.001\right.$; Figure 4$)$ in our pooled sample. Despair ratings were higher in female listeners, independently from the context $(\beta \pm S E=-$ $0.368 \pm 0.124 ; z=-2.973 ; p=.003$ ), while, in the other scales, we found no gender differences in the ratings (aggression: $\beta \pm S E=-0.059 \pm 0.122 ; z=-0.489 ; p=.625$; fear: $\beta \pm S E=-0.046 \pm 0.121 ; z=-0.382 ; p=$ .703 ; play: $\beta \pm S E=0.054 \pm 0.115 ; z=0.469 ; p=.639$; happy: $\beta \pm S E=0.043 \pm 0.112 ; z=0.387 ; p=$ $.699)$.

\section{Categorization of Bark Samples}

\section{Replication of Context Categorization}

We found that, in the Portuguese sample, both the bark context $\left(L R T: \chi^{2}(5)=63.84 ; p<.001\right)$ and the ownership status $\left(L R T: \chi^{2}(1)=7.18 ; p=.007\right)$ had a significant effect on context categorization. Posthoc comparisons (Figure 5; Table S1D) showed that listeners recognized 'stranger at the fence' barks the most, while 'going for walk' and 'ball' were the most difficult contexts to recognize. The 'alone,' 'play,' and 'Schutzhund' contexts were recognized at an intermediate level.

Also, we found that Portuguese dog owners were more successful at recognizing the context of the barks than the participants from this country who have never owned a $\operatorname{dog}(O R=1.553,95 \% C I[1.135-$ 2.124]; $z=2.754 ; p=.006$; Figure 6). 
Silva et al. 114

\section{Figure 4}

Interaction Between the Gender of the Listeners and the Emotional Scales

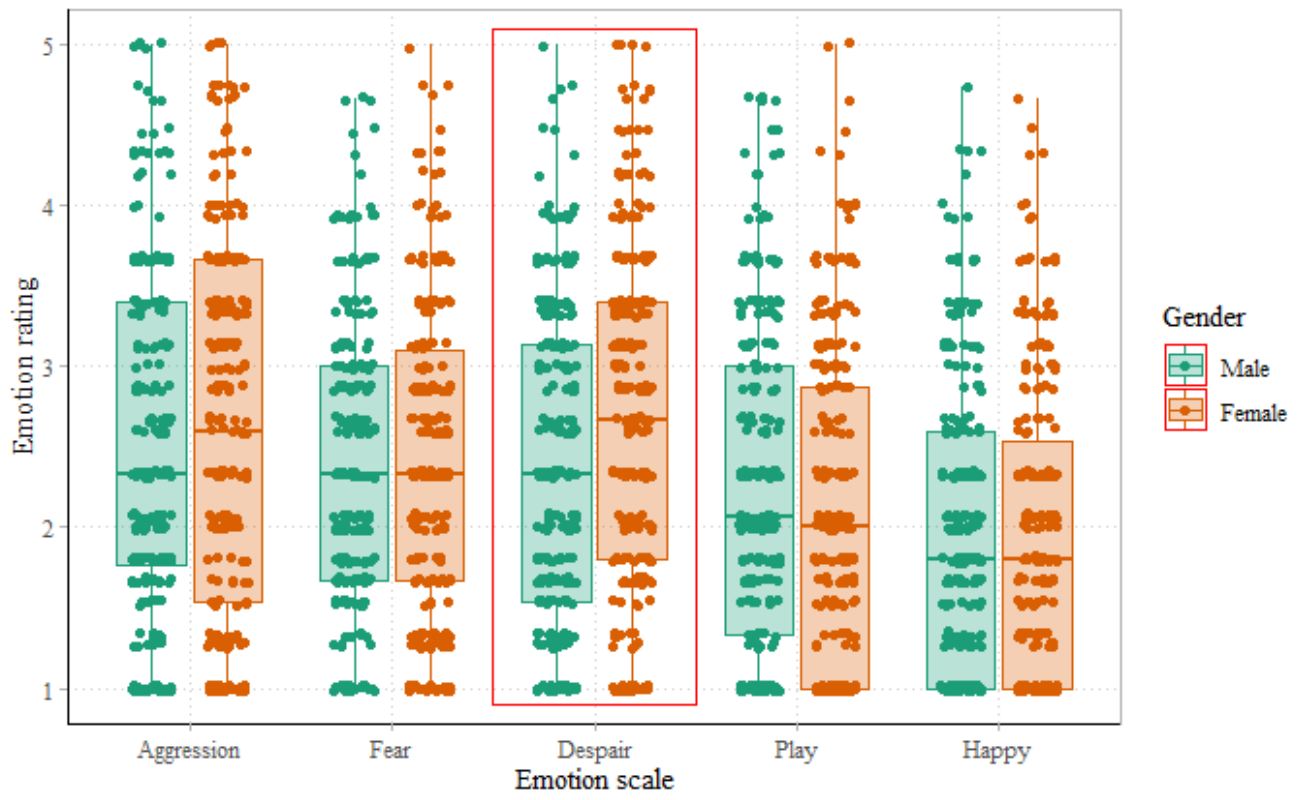

Note. Women tend to rate the overall barks significantly higher on the despair scale. Within the boxplots the horizontal line represents the median, the box shows the quartiles, the whiskers the range while the circles the outliers. The red rectangle shows the significant difference.

Figure 5

Difference Between the Recognition Success in the Different Contexts

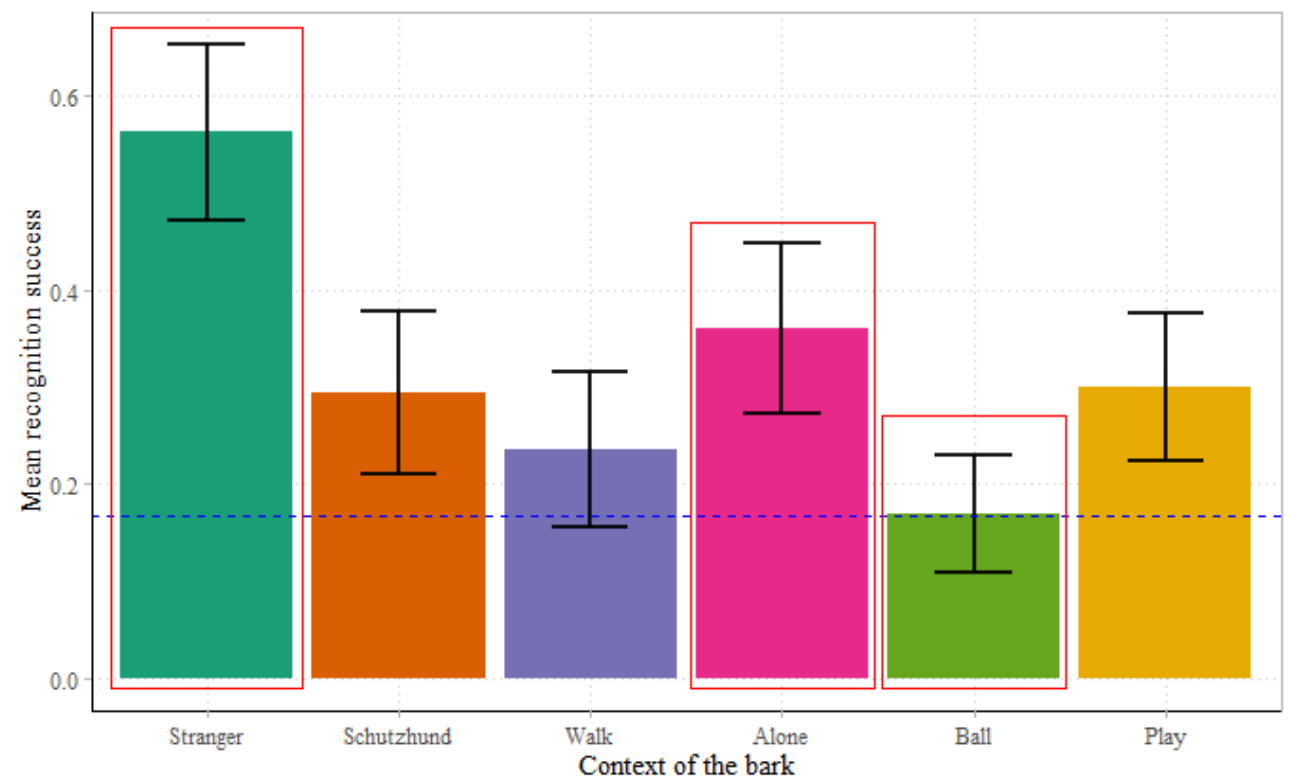

Note. Success rate was the highest in the 'stranger' context, while the lowest in the 'walk' and 'ball' contexts. In the case of 'Schutzhund,' 'walk' and 'play' the subjects' performance did not differ from the chance level (.167), and in the 'ball' context they performed significantly below the chance level. The bars show the mean ratio of correct answers with standard error. The red rectangles indicate a significant difference from chance. 


\section{Figure 6}

\section{The Effect of Experience on Context Recognition}

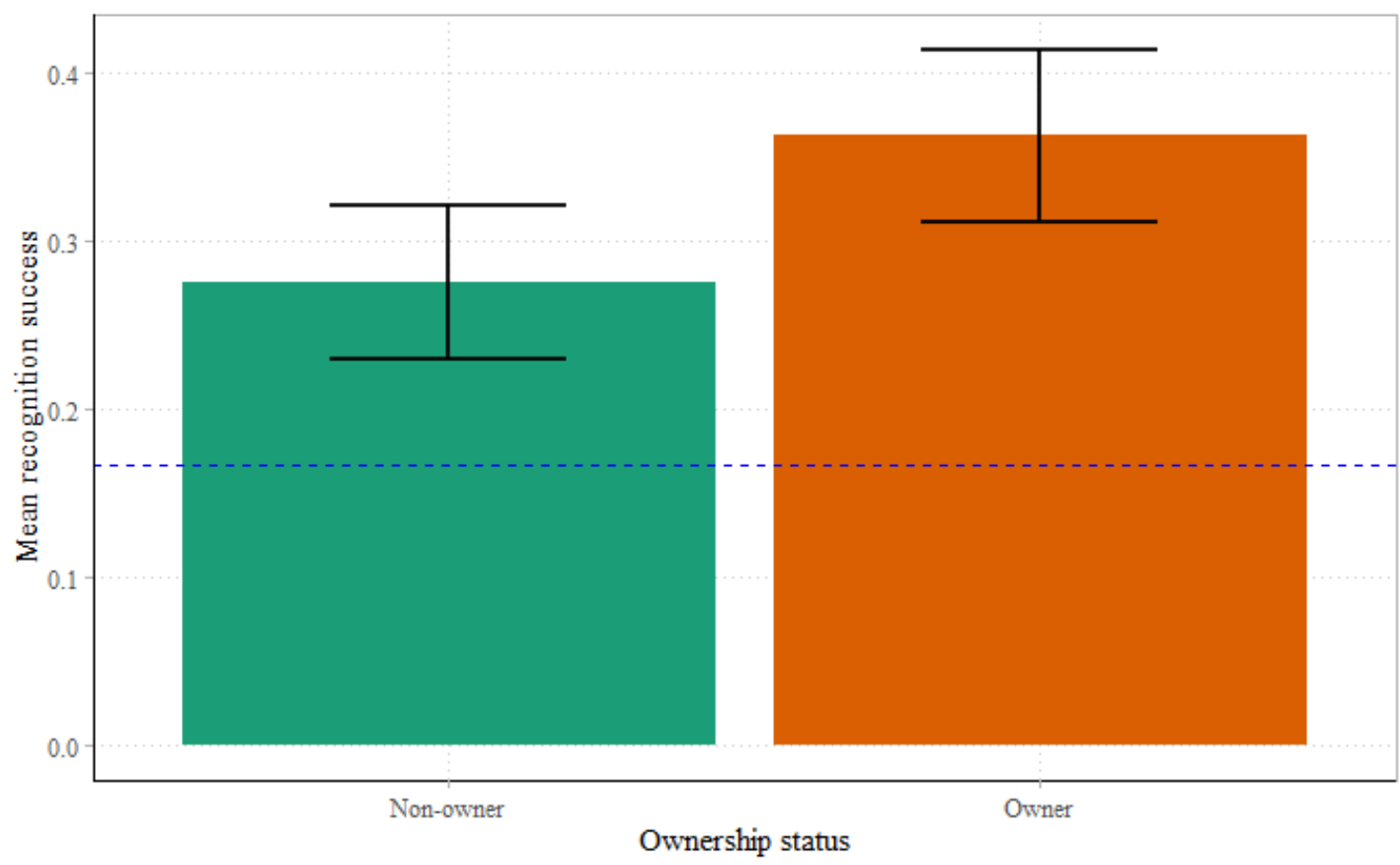

Note. Owners had higher success in recognition of the bark contexts compared to non-owners. The error bars show the SE.

Regarding the within context success, one-sample Wilcoxon signed rank tests showed recognition accuracy above chance level for two contexts (Table 1): stranger and alone barks. In case of the 'Schutzhund', 'walk' and play barks, no difference from chance level was found, while the 'ball' barks were recognized significantly under chance level by the Portuguese listeners.

Table 1

Recognition Success of the Six Contexts from Which Barks Were Recorded

\begin{tabular}{ccc}
\hline Context & W & $p$ \\
\hline Stranger & 1207 & $<.001$ \\
Schutzhund & 738 & $=.479$ \\
Walk & 559 & $=.322$ \\
Alone & 901 & $=.024$ \\
Ball & 369 & $=.005$ \\
Play & 794 & $=.215$ \\
\hline
\end{tabular}

Note. The table shows the details of the one-sample Wilcoxon signed rank tests against the random choice ( $16.7 \%$ success).

\section{Difference Between the Portuguese and Hungarian Success Levels of Context Recognition}

We found a significant difference between the success rate of the Hungarians and Portuguese participants $\left(L R T: \chi^{2}(1)=8.91 ; p=.003\right)$, with an advantage of Hungarians $(O R=0.693,95 \%$ CI $[0.548$ $0.877] ; z=-3.051 ; p=.002$; Figure 7). 


\section{Figure 7}

The Difference Between the Two Countries in Recognition Success

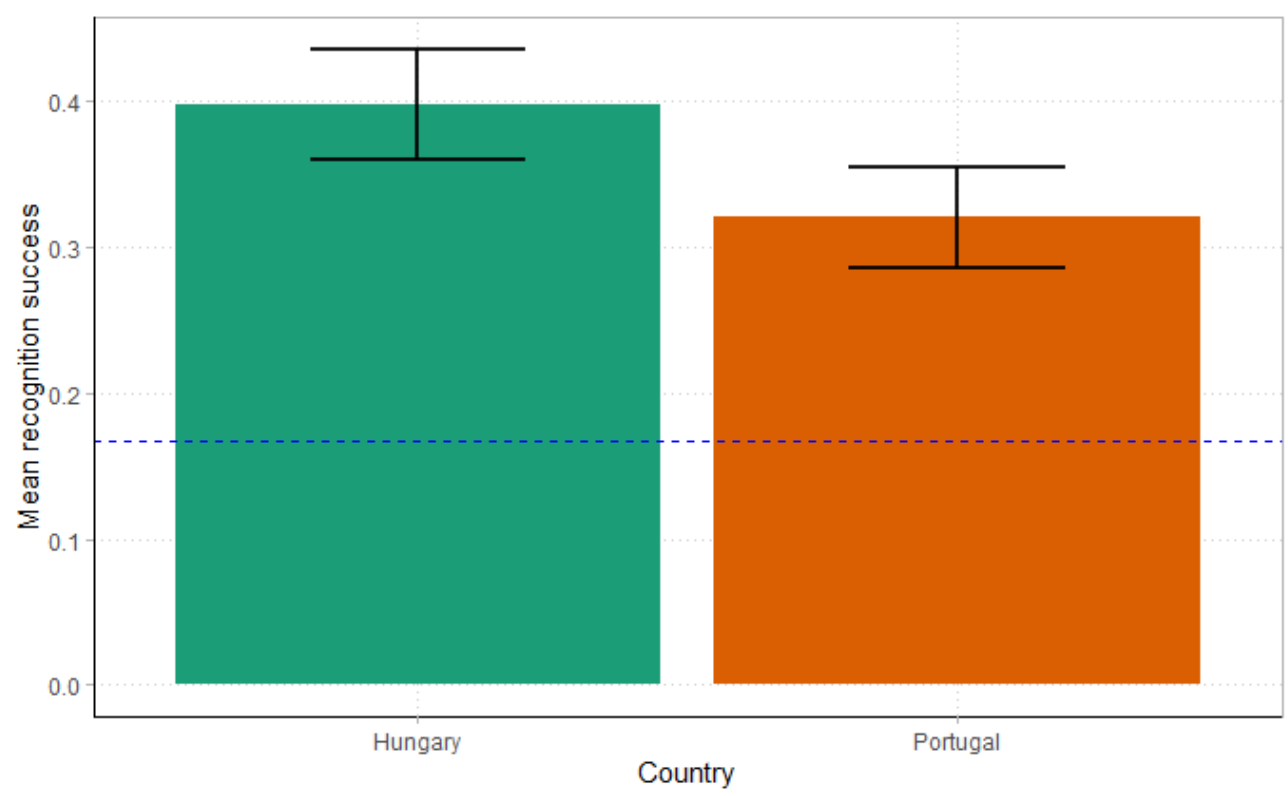

Note. Hungarian participants were more successful guessing the barks' social context. The error bars show the SE.

\section{Gender Effect}

We found no difference in context recognition success between male and female participants in the pooled sample.

\section{Error Pattern of the Context Recognition}

As a significant difference was found in context recognition accuracy between residents of the two countries, one could ask whether this difference was due to a difference in participant error patterns or to a generally lower recognition accuracy in the Portuguese sample. For this purpose, a so-called 'confusion' matrix was generated separately for each group of subjects. As can be seen in Table 2, the error pattern was similar between the two countries with a lower general accuracy in the Portuguese sample.

Most of the barks from the 'stranger' context were correctly recognized in both countries, while the barks from the 'Schutzhund' context were mainly confused with the similarly agonistic 'stranger' context. It is worth noting here that Portuguese listeners assigned almost half of the 'Schutzhund' barks to the 'stranger' category. In respect to the barks from the 'play' context, Hungarian listeners mostly confused them with barks from the 'ball' context. In Portugal, these were also often assigned to the 'Schutzhund' context. In case of the 'before walk' and the 'ball' contexts, the recognition accuracy was low and the errors were evenly distributed among the other contexts (except the 'Schutzhund') in both countries. In case of barks from the 'alone' context, the Hungarians categorized almost half of the samples correctly, and the rest were evenly distributed. Portuguese listeners confused this context mainly with the 'before walk' and the 'stranger' contexts.

Table 2 


\begin{tabular}{|c|c|c|c|c|c|c|c|c|}
\hline \multirow{2}{*}{\multicolumn{3}{|c|}{ Percentage of responses }} & \multicolumn{6}{|c|}{ Contexts } \\
\hline & 更 & Destist & Stranger & Schutzhund & Walk & Alone & Ball & Play \\
\hline \multirow{12}{*}{ 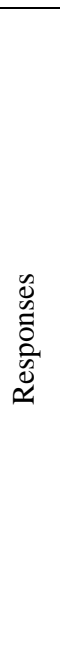 } & \multirow{6}{*}{ 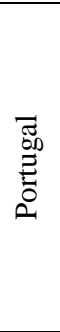 } & Stranger & 56.86 & 37.91 & 16.34 & 15.69 & 24.19 & 4.58 \\
\hline & & Schutzhund & 3.27 & 29.41 & 6.54 & 3.92 & 1.96 & 26.80 \\
\hline & & Walk & 7.19 & 7.19 & 23.53 & 22.22 & 11.11 & 11.76 \\
\hline & & Alone & 14.38 & 6.54 & 25.49 & 35.95 & 28.76 & 7.18 \\
\hline & & Ball & 11.11 & 12.42 & 11.76 & 9.80 & 16.99 & 19.61 \\
\hline & & Play & 7.19 & 5.88 & 16.34 & 12.42 & 16.99 & 30.07 \\
\hline & \multirow{6}{*}{ 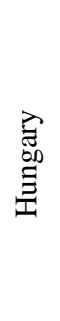 } & Stranger & 58.33 & 30.56 & 11.11 & 12.96 & 16.67 & 4.63 \\
\hline & & Schutzhund & 12.96 & 48.15 & 4.63 & 4.63 & 6.48 & 12.96 \\
\hline & & Walk & 7.41 & 3.70 & 23.15 & 10.19 & 11.11 & 12.96 \\
\hline & & Alone & 9.26 & 4.63 & 18.52 & 47.22 & 25.93 & 9.26 \\
\hline & & Ball & 5.56 & 3.70 & 20.37 & 14.81 & 25.00 & 23.15 \\
\hline & & Play & 6.48 & 9.26 & 22.22 & 10.19 & 14.81 & 37.04 \\
\hline
\end{tabular}

Note. The cells contain the percentage of the corresponding answers. The numbers highlighted with bold show the correct choices.

\section{Discussion}

The aim of the present study was to provide a replication of Pongrácz et al.'s (2005) study as a first and preliminary step into research exploring the possibility of cross-cultural differences in humans' ability to perceive and understand dog barks. Our findings add to the existing literature in that they suggest that both universal (i.e., biological) and country/culture-specific characteristics modulate the perception and understanding of dogs' emotional vocalizations by humans.

Similar to the Hungarians (as reported in Pongrácz et al., 2005), Portuguese participants in the present study rated dogs' barks in accordance with expectations on how the specific social contexts could affect the inner states of dogs (for example, vocalizations of dogs encountering a stranger in the garden were given high scores of aggressiveness and low scores of playfulness). Although less effectively than the Hungarians, Portuguese listeners categorized two of the bark samples recorded from different contexts above chance level. A similar error pattern was observed across the two groups of listeners, showing that they confused roughly the same pairs of contexts with each other. Such similarities between Portuguese and Hungarian listeners are in line with previous observations suggesting that dog vocalizations are familiar and informative to humans in general (e.g., Pongrácz et al., 2010). Moreover, the observed similarities are in accordance with theories of shared emotional systems across mammalian species (first postulated by Darwin (1965), and later described for emotional vocalizations in Morton's Motivation Structural Rules (Morton, 1977), suggesting the background mechanism of simple emotion encoding rules (Andics \& Faragó, 2019; Faragó et al., 2014; Filippi et al., 2017) within barks too. Besides the reactions to alarm, mobbing or food calls, between-species emotional decoding in other contexts is lately in the forefront of comparative cognitive research. Bi-directional information transfer was detected between humans and various domesticated species. Among others, it was also found that humans use dog-specific prosody and acoustic cues when they verbally address dogs (Gergely et al., 2017) and these cues affect the attentional state of the dogs (Jeannin, Gilbert, \& Leboucher, 2017; Jeannin, Gilbert, Amy et al., 2017). Dogs are also exceptionally sensitive to human emotional cues (Albuquerque et al., 2016; Müller et al., 2015; Turcsán et al., 2015). Moreover, a recent study has shown that, not just humans but even birds, are capable of 
perceiving emotion intensity in various, evolutionarily distant species' calls (Congdon et al., 2019). All these examples, in line with our findings strongly support the importance of biologically rooted, simple rules of vocal emotion encoding.

Results, however, also unveiled significant differences between the data obtained here and that previously reported by Pongrácz et al. (2005) regarding both the emotionality ratings attributed to the bark samples and their categorization. Portuguese participants in this study tended to perceive the agonistic barks ('stranger' and 'Schutzhund') to be less aggressive, but 'play' barks as being more aggressive than as it was found in the case of the Hungarians. They rated barks from the 'before walk' context more negatively (more fearful and desperate and less playful and happy) than Hungarian subjects tested in Pongrácz et al. (2005). Also, Portuguese listeners tended to attribute higher levels of despair to the 'Schutzhund' barks (almost as high as on aggressiveness) than the Hungarians did. Hungarian listeners, in turn, were more successful in categorizing the bark samples.

The observed pattern of results suggests that context recognition performance may have been affected primarily by the perception of emotion in the barks: Portuguese listeners tended to consider the barks to be more negative and less playful (perhaps due to a country-specific negativity bias, Chang et al., 2001; Vaish et al., 2008). Previous research from Pongrácz and colleagues (2005) also highlighted the fact that humans tend to categorize dog barks through responding to the emotional content of them at first and then 'translating' this information to a plausible contextual scenario. Similarly, in a study assessing human decoding of piglet (Sus scrofa domestica) calls, Maruščáková et al. (2015) showed that perceived emotional intensity guided listeners' identification of the purported eliciting context. More specifically, the probability of correct context recognition was positively associated with the assessed emotion intensity for some calls (castration and reunion calls), and negatively for others (nursing calls).

According to Marušćáková et al. (2015), one potential mechanism by which perceived animal vocalizations' emotional intensity might be used by humans to identify the associated context of such vocalizations is that listeners might have a preconception of the emotional impact/intensity of the different situations. As also highlighted by these authors, similar mechanisms seem to be at work when human adults decode vocalizations of preverbal children (e.g., Lindová et al., 2015). Thus, it may be that the Hungarians' higher accuracy in categorizing the bark samples might have been guided by more accurate preconceptions of the emotional nature of dogs, in general, and of the different bark contexts, in particular. Such differences in preconceptions between Portuguese people and Hungarians, in turn, could originate from differences in personal experience with similar situations, either involving Mudis or dogs in general, and/or differences in attitudes towards dogs. To this point, however, this hypothesis is only speculative and further research is needed to explore the potential effects of these factors with direct comparisons of attitudes towards dogs and dog barking or playing back a Portuguese breed's barks to participants from both countries.

It seems important to note that only presumptions can be made about the inner state of the dogs in a given context. We cannot say, therefore, that Portuguese subjects interpreted or read dogs' emotion worse than the Hungarians (and vice versa) as it is possible that the actual emotional state of the dogs can be interpreted in line with the Portuguese subjects' perception (for example, as fear-aggression towards an attacking human in the 'Schutzhund' context). In other words, barks' acoustic structure reflects the dogs' complex inner state and listeners from different cultures might well perceive different aspects to be more salient due to their experiences and cultural 'filters' (including, for instance, the country-specific negativity bias mentioned above; Chang et al., 2001; Vaish et al., 2008) leading to differences in interpretation.

The results also showed that women from both countries tended to attribute higher levels of despair to all the bark samples. Furthermore, dog owners were significantly more accurate in categorizing the bark samples than all the other listeners. Such observations are of particular interest because, to our knowledge, this is the first study providing data that show that the perception of an emotion in dogs using auditory cues of dog barks varies by experience and sex. Despite evidence that neural activity in response to dog behavior varies according to experience with dogs (Kujala et al., 2012), previous studies found only limited support for the effect of this factor on the perception of emotion in dog vocalizations (Faragó et al., 2014; Pongrácz et al., 2005). More recently, it was found that, in recognition of the context of growls, women and dog owners had an advantage, but no difference was found in emotion ratings (Faragó et al., 2017). However, 
whereas barks are loud and salient vocalizations that are hard to ignore, growls are short distance calls used in close interaction, thus experience can have a stronger effect in their recognition. Although some studies on children found that the accurate decoding of emotions in dog barks increased with age, it remained unclear whether this was due to cumulative experience with dogs or the development of emotion processing systems (Pongrácz et al., 2011). Moreover, in studies with adult participants, neither visual experience nor experience with dogs predicted accuracy in the interpretation of dog barks (Molnár et al., 2010).

The understanding of another human's emotional nonverbal signs depends, at least to some degree, on empathy (Dziobek et al., 2008). In human studies, and as highlighted in Maruščáková et al. (2015), a distinction is often made between cognitive empathy and emotional empathy. The former is commonly defined as the capacity of individuals to take the perspective of others (without the necessity of sharing the same emotional state), and the latter as the emotional response to the emotional state of another person (Dziobek et al., 2008). Interestingly, several studies point to gender differences in empathy with men relying more heavily on their cognitive-emotional control and perspective taking than women (e.g., Schulte-Rüther et al., 2008). Based on this, one could entertain the hypothesis that higher cognitive empathy along with greater experience with dogs might have enabled male owners to judge more precisely the situational context in which the barks were emitted. In turn, potentially higher levels of emotional empathy of women might explain the higher ratings of despair attributed to all the bark samples. Accordingly, previous studies have shown that women tend to become more emotionally involved with their pets and are more likely than men to regard them as family members (Selby \& Rhoades, 1981) while other studies suggest a negativitybias linked with higher level of empathy, that can explain our findings (Chikovani et al., 2015). According to Miura et al. (2000), one of the possible explanations for these gender differences would be a tendency that "women are socialized from birth for nurturing and caring roles while men are trained to be less emotional and more utilitarian" (Herzog Jr. et al., 1991, p. 190).

\section{Limitations}

Our study has some limitations, partly stemming from being a replication of an earlier investigation, and partly because the shortcomings of the original testing paradigm. Starting with the latter, emotional scoring can be considered as only an approximation of the subjective perception of the inner state of the signaler, as we do not have empirical test results of the actual inner states of the barking dogs. Opting for the sound samples of a single dog breed provided the advantage of eliminating a large contingent of acoustic variability of the barks; however, this may warrant further studies where the validity of present and former findings could be cross-checked with other dog breeds' results. Some limitations of the replication process itself are, for instance, that as there were more than 10 years between the two studies, this amount of time could be relevant in changing attitudes toward dogs in general - which in turn, could affect the humans' expectations about dogs' communicative capabilities and their empathetic approach towards dogs. The replication was also lacking the group of Mudi owners in Portugal (due to the rarity of this dog breed) and detailed background information about the participants in the original study making an exact match of samples impossible.

\section{Conclusion}

More than a decade after the original paper of Pongrácz et al. (2005), the present study is the first to present a cross-cultural examination of humans' ability to perceive and understand dog barks. Results show that human decoding of the emotional and contextual content of dog barks presents some level of cross-cultural robustness. Indeed, we found that the vocal signals (barks) of an originally randomly chosen dog breed, the Mudi, convey information that is perceived with high level of similarity by Portuguese and Hungarian listeners. This result provides indirect evidence that dog barks, independent of the familiarity with the breed, may serve as an equally effective source of information for humans. Additionally, and beyond the general match between the performance of the Portuguese and the Hungarian listeners, our 
results also highlighted some interesting differences between the two countries, which warrant the possible effect of cross-country variability of emotional perception and open the way for further investigations comparing more culturally different countries (e.g., Easterners versus Westerners countries; Chang et al., 2001). Moreover, they unveil interesting effects of gender and the dog-related experiences of the human participants. Future research is recommended to bring light into the exact processes underlying such an interesting pattern of results highlighting both culture dependent and independent factors affecting human's ability to categorize dog barks. Also, a broader investigation of the universality of emotional and contextual information encoded in dog barks would be useful by the involvement of a wider selection of dog breeds, preferably with different genetic background and also a different history of purpose-related selection. Also, a wider comparison of different cultures with markedly different attitudes (e.g., regarding dogs as unclean) and experiences (e.g., keeping dogs as pets, working partners or having only pariah dogs around) with dogs might be fruitful to broaden our knowledge about dog-human communication.

\section{Acknowledgments}

The authors want to thank Romain Piault for his valuable comments on a preliminary version of this paper and for Nora Bunford for checking the language of the manuscript.

\section{Ethical Statement}

All procedures performed involving human participants were in accordance with the ethical standards of the institutional and/or national research committee and with the 1964 Helsinki declaration and its later amendments or comparable ethical standards. Participation was voluntary and all subjects were informed about the aim of the study and the procedure. All personal data was handled anonymously and confidentially. Ethical approval was provided by the United Psychological Research Ethics Committee (EPKEB 2016/003).

\section{Funding Statement}

Fundação para a Ciência e a Tecnologia funded the participation of KS (SFRH/BPD/100556/2014). PP was funded by the Hungarian Ministry of Education, OTKA K82020. TF was funded by the Hungarian Academy of Sciences, MTA (01 031) and by the Office for Research Groups Attached to Universities and Other Institutions of the Hungarian Academy of Sciences in Hungary, MTA Premium Post Doctorate Grant (460002), the European Research Council (ERC) under the European Union's Horizon 2020 research and innovation programme (680040), the János Bolyai Research Scholarship of the Hungarian Academy of

Sciences (BO/751/20), the New National Excellence Program of the Ministry for Innovation and Technology (ÚNKP-20-5-ELTE-337).

\section{Data Accessibility}

Data is available here: http://animalbehaviorandcognition.org/uploads/files/Silva_Farago_etal $\mathrm{ABC} \quad 8 \quad 2 \quad$ Table-S1.xls

\section{Competing Interests}

The authors report no conflicts of interest.

\section{Authors' Contributions}


KS contributed to conception and study design, acquisition of data, interpretation of data, drafting and correction of the manuscript.

TF contributed to study design, statistical analysis, interpretation of data, drafting and correction of the manuscript.

PP contributed to study design, drafting and correction of the manuscript.

PR contributed to study design and acquisition of data.

ML contributed to study design, drafting and revision of the manuscript.

LdS contributed to study conception and revision of the manuscript.

\section{References}

Albuquerque, N., Guo, K., Wilkinson, A., Savalli, C., Otta, E., \& Mills, D. S. (2016). Dogs recognize dog and human emotions. Biology Letters, 12(1), 20150883. https://doi.org/10.1098/rsbl.2015.0883

Andics, A., \& Faragó, T. (2019). Voice perception across species. In S. Frühholz \& P. Belin (Eds.), The Oxford handbook of voice perception (1st ed., pp. 362-392). Oxford University Press. https://doi.org/10.1093/oxfordhb/9780198743187.013.16

Andics, A., Gácsi, M., Faragó, T., Kis, A., \& Miklósi, Á. (2014). Voice-sensitive regions in the dog and human brain are revealed by comparative fMRI. Current Biology, 24(5), 574-578. https://doi.org/10.1016/j.cub.2014.01.058

Chang, E. C., Asakawa, K., \& Sanna, L. J. (2001). Cultural variations in optimistic and pessimistic bias: Do Easterners really expect the worst and Westerners really expect the best when predicting future life events? Journal of Personality and Social Psychology, 81(3), 476-491. https://doi.org/10.1037/0022-3514.81.3.476

Chikovani, G., Babuadze, L., Iashvili, N., Gvalia, T., \& Surguladze, S. (2015). Empathy costs: Negative emotional bias in high empathisers. Psychiatry Research, 229(1-2), 340-346. https://doi.org/10.1016/j.psychres.2015.07.001

Congdon, J. V., Hahn, A. H., Filippi, P., Campbell, K. A., Hoang, J., Scully, E. N., Bowling, D. L., Reber, S. A., \& Sturdy, C. B. (2019). Hear them roar: A comparison of black-capped chickadee (Poecile atricapillus) and human (Homo sapiens) perception of arousal in vocalizations across all classes of terrestrial vertebrates. Journal of Comparative Psychology, 133(4), 520-541. https://doi.org/10.1037/com0000187

Darwin, C. (1965). The expression of the emotions in man and animals (1872). University of Chicago Press.

Dziobek, I., Rogers, K., Fleck, S., Bahnemann, M., Heekeren, H. R., Wolf, O. T., \& Convit, A. (2008). Dissociation of cognitive and emotional empathy in adults with Asperger syndrome using the Multifaceted Empathy Test (MET). Journal of Autism and Developmental Disorders, 38(3), 464-473. https://doi.org/10.1007/s10803-007-0486-X

Ekman, P., Friesen, W. V., O’Sullivan, M., Chan, A., Diacoyanni-Tarlatzis, I., Heider, K., Krause, R., LeCompte, W. A., Pitcairn, T., Ricci-Bitti, P. E., Scherer, K., Tomita, M., \& Tsavaras, A.(1987). Universals and cultural differences in the judgments of facial expressions of emotion. Journal of Personality and Social Psychology, 53(4), 712-717. https://doi.org/10.1037/0022-3514.53.4.712

Elfenbein, H. A. (2006). Learning in emotion judgments: Training and the cross-cultural understanding of facial expressions. Journal of Nonverbal Behavior, 30(1), 21-36. https://doi.org/10.1007/s10919-005-0002-y

Elfenbein, H. A., \& Ambady, N. (2002). On the universality and cultural specificity of emotion recognition: A metaanalysis. Psychological Bulletin, 128(2), 203-235. https://doi.org/10.1037//0033-2909.128.2.203

Elfenbein, H. A., Mandal, M. K., Ambady, N., Harizuka, S., \& Kumar, S. (2002). Cross-cultural patterns in emotion recognition: Highlighting design and analytical techniques. Emotion, 2(1), 75-84. https://doi.org/10.1037/1528-3542.2.1.75

Fallow, P. M., \& Magrath, R. D. (2010). Eavesdropping on other species: Mutual interspecific understanding of urgency information in avian alarm calls. Animal Behaviour, 79(2), 411-417. https://doi.org/10.1016/j.anbehav.2009.11.018

Faragó, T., Andics, A., Devecseri, V., Kis, A., Gácsi, M., \& Miklósi, Á. (2014). Humans rely on the same rules to assess emotional valence and intensity in conspecific and dog vocalizations. Biology Letters, 10(1), 20130926. https://doi.org/10.1098/rsbl.2013.0926

Faragó, T., Takács, N., Miklósi, Á., \& Pongrácz, P. (2017). Dog growls express various contextual and affective content for human listeners. Royal Society Open Science, 4(5), 170134. https://doi.org/10.1098/rsos.170134

Filippi, P., Congdon, J. V., Hoang, J., Bowling, D. L., Reber, S. A., Pašukonis, A., Hoeschele, M., Ocklenburg, S., de Boer, B., Sturdy, C. B., Newen, A., \& Güntürkün, O. (2017). Humans recognize emotional arousal in 
vocalizations across all classes of terrestrial vertebrates: Evidence for acoustic universals. Proceedings of the Royal Society B: Biological Sciences, 284(1859), 20170990. https://doi.org/10.1098/rspb.2017.0990

Gergely, A., Faragó, T., Galambos, Á., \& Topál, J. (2017). Differential effects of speech situations on mothers' and fathers' infant-directed and dog-directed speech: An acoustic analysis. Scientific Reports, 7(1), 13739. https://doi.org/10.1038/s41598-017-13883-2

Herzog Jr., H. A., Betchart, N. S., \& Pittman, R. B. (1991). Gender, sex role orientation, and attitudes toward animals. Anthrozoös, 4(3), 184-191. https://doi.org/10.2752/089279391787057170

Jeannin, S., Gilbert, C., Amy, M., \& Leboucher, G. (2017). Pet-directed speech draws adult dogs' attention more efficiently than Adult-directed speech. Scientific Reports, 7(1), 4980. https://doi.org/10.1038/s41598-017$\underline{04671-\mathrm{Z}}$

Jeannin, S., Gilbert, C., \& Leboucher, G. (2017). Effect of interaction type on the characteristics of pet-directed speech in female dog owners. Animal Cognition, 20(3), 499-509. https://doi.org/10.1007/s10071-017$\underline{1077-7}$

Kitchen, D. M., Bergman, T. J., Cheney, D. L., Nicholson, J. R., \& Seyfarth, R. M. (2010). Comparing responses of four ungulate species to playbacks of baboon alarm calls. Animal Cognition, 13(6), 861-870. https://doi.org/10.1007/s10071-010-0334-9

Koda, H. (2012). Possible use of heterospecific food-associated calls of macaques by sika deer for foraging efficiency. Behavioural Processes, 91(1), 30-34. https://doi.org/10.1016/j.beproc.2012.05.006

Kujala, M. V, Kujala, J., Carlson, S., \& Hari, R. (2012). Dog experts' brains distinguish socially relevant body postures similarly in dogs and humans. PloS One, 7(6), e39145. https://doi.org/10.1371/journal.pone.0039145

Lindová, J., Špinka, M., \& Nováková, L. (2015). Decoding of baby calls: Can adult humans identify the eliciting situation from emotional vocalizations of preverbal infants? PLOS ONE, 1O(4), e0124317. https://doi.org/10.1371/journal.pone.0124317

Linnankoski, I., Laakso, M.-L., Aulanko, R., \& Leinonen, L. (1994). Recognition of emotions in macaque vocalizations by children and adults. Language \& Communication, 14(2), 183-192. https://doi.org/10.1016/0271-5309(94)90012-4

Magrath, R. D., Haff, T. M., Fallow, P. M., \& Radford, A. N. (2015). Eavesdropping on heterospecific alarm calls: From mechanisms to consequences. Biological Reviews, 90(2), 560-586. https://doi.org/10.1111/brv.12122

Maruščáková, I. L., Linhart, P., Ratcliffe, V. F., Tallet, C., Reby, D., \& Špinka, M. (2015). Humans (Homo sapiens) judge the emotional content of piglet (Sus scrofa domestica) calls based on simple acoustic parameters, not personality, empathy, nor attitude toward animals. Journal of Comparative Psychology, 129(2), 121-131. https://doi.org/10.1037/a0038870

Matsumoto, D., \& Assar, M. (1992). The effects of language on judgments of universal facial expressions of emotion. Journal of Nonverbal Behavior, 16(2), 85-99. https://doi.org/10.1007/BF00990324

Matsumoto, D., \& Hwang, H. S. (2012). Culture and emotion: The integration of biological and cultural contributions. Journal of Cross-Cultural Psychology, 43(1), 91-118. https://doi.org/10.1177/0022022111420147

Miura, A., Bradshaw, J. W. S., \& Tanida, H. (2000). Attitudes towards dogs: A study of university students in Japan and the UK. Anthrozoos, 13(2), 80-88. https://doi.org/10.2752/089279300786999860

Molnár, C., Pongrácz, P., \& Miklósi, Á. (2010). Seeing with ears: Sightless humans’ perception of dog bark provides a test for structural rules in vocal communication. Quarterly Journal of Experimental Psychology, 63(5), 1004-1013. https://doi.org/10.1080/17470210903168243

Morton, E. S. (1977). On the occurrence and significance of motivation - structural rules in some bird and mammal sounds. The American Naturalist, 111 (981), 855-869. https://doi.org/10.1086/283219

Müller, C. A., Schmitt, K., Barber, A. L. A., \& Huber, L. (2015). Dogs can discriminate emotional expressions of human faces. Current Biology, 25(5) 601-605. https://doi.org/10.1016/j.cub.2014.12.055

Nicastro, N., \& Owren, M. J. (2003). Classification of domestic cat (Felis catus) vocalizations by naive and experienced human listeners. Journal of Comparative Psychology, 117(1), 44-52. https://doi.org/10.1037/0735-7036.117.1.44

Pongrácz, P. (2017). Modeling evolutionary changes in the information transfer - effects of domestication on the vocal communication of dogs (Canis familiaris). European Psychologist, 22(4), 219-232. https://doi.org/10.1027/1016-9040/a000300

Pongrácz, P., Molnár, C., Dóka, A., \& Miklósi, Á. (2011). Do children understand man’s best friend? Classification of dog barks by pre-adolescents and adults. Applied Animal Behaviour Science, 135(1-2), 95-102. https://doi.org/10.1016/j.applanim.2011.09.005 
Pongrácz, P., Molnár, C., \& Miklósi, Á. (2006). Acoustic parameters of dog barks carry emotional information for humans. Applied Animal Behaviour Science, 100(3-4), 228-240. https://doi.org/10.1016/j.applanim.2005.12.004

Pongrácz, P., Molnár, C., \& Miklósi, Á. (2010). Barking in family dogs: An ethological approach. The Veterinary Journal, 183(2), 141-147. https://doi.org/10.1016/j.tvjl.2008.12.010

Pongrácz, P., Molnár, C., Miklósi, Á., \& Csányi, V. (2005). Human listeners are able to classify dog (Canis familiaris) barks recorded in different situations. Journal of Comparative Psychology, 119(2), 136-144. https://doi.org/10.1037/0735-7036.119.2.136

Scherer, K. R. (1997). The role of culture in emotion-antecedent appraisal. Journal of Personality and Social Psychology, 73(5), 902-922. https://doi.org/10.1037/0022-3514.73.5.902

Schulte-Rüther, M., Markowitsch, H. J., Shah, N. J., Fink, G. R., \& Piefke, M. (2008). Gender differences in brain networks supporting empathy. NeuroImage, 42(1), 393-403. https://doi.org/10.1016/j.neuroimage.2008.04.180

Selby, L. A., \& Rhoades, J. D. (1981). Attitudes of the public towards dogs and cats as companion animals. Journal of Small Animal Practice, 22(3), 129-137. https://doi.org/10.1111/j.1748-5827.1981.tb00592.x

Shao, B., Doucet, L., \& Caruso, D. R. (2014). Universality versus cultural specificity of three emotion domains: Some evidence based on the cascading model of emotional intelligence. Journal of Cross-Cultural Psychology, 46(2), 229-251. https://doi.org/10.1177/0022022114557479

Tallet, C., Špinka, M., Marušćáková, I. L., \& Šimeček, P. (2010). Human perception of vocalizations of domestic piglets and modulation by experience with domestic pigs (Sus scrofa). Journal of Comparative Psychology, 124(1), 81-91. https://doi.org/10.1037/a0017354

Turcsán, B., Range, F., Virányi, Z., Miklósi, Á., \& Kubinyi, E. (2012). Birds of a feather flock together? Perceived personality matching in owner-dog dyads. Applied Animal Behaviour Science, 140(3-4), 154-160. https://doi.org/10.1016/j.applanim.2012.06.004

Turcsán, B., Szánthó, F., Miklósi, Á., \& Kubinyi, E. (2015). Fetching what the owner prefers? Dogs recognize disgust and happiness in human behaviour. Animal Cognition, 18(1), 83-94. https://doi.org/10.1007/s10071-014-0779-3

Vaish, A., Grossmann, T., \& Woodward, A. (2008). Not all emotions are created equal: The negativity bias in social-emotional development. Psychological Bulletin, 134(3), 383-403. https://doi.org/10.1037/0033$\underline{2909.134 .3 .383}$ 